Mathematical Research Letters 8, 25-33 (2001)

\title{
HIGHER-DIMENSIONAL PARALLEL TRANSPORTS
}

\author{
KiYOnori Gomi and Yuji Terashima*
}

\section{Introduction}

For a smooth path $\gamma$ in a manifold $X$, a connection on a line bundle over $X$ gives an isomorphism between the fibers called the parallel transport along $\gamma$. In this paper, viewing a path as a map from a 1-dimensional manifold with boundary to $X$ and a line bundle with connection as a 1-dimensional smooth Deligne cocycle of $X([\mathrm{D}],[\mathrm{Ko}])$, we generalize such a concept to maps from a higher-dimensional manifold with boundary to $X$ and a higher-dimensional smooth Deligne cocycle. Firstly we use transgression maps on smooth Deligne cochains to construct line bundles with connection over the spaces of maps from closed manifolds of dimension $m$ to $X$ for a smooth Deligne cocycle of degree $m+1$. Secondly for a compact manifold $N$ of dimension $m+1$ with boundary we get a global non-vanishing section of the line bundle over the space of maps from $N$ to $X$, which is the pullback by the restriction maps of the line bundles associated with the closed $m$-manifolds of the boundary components. Then, for each map $f$ from $N$ to $X$ the section gives the isomorphism between the fibers, called the higher-dimensional parallel transport along $f$. Finally we prove that for $f: N=M \times[0,1] \rightarrow X$, where $M$ is a closed manifold of dimension $m$, the higher dimensional parallel transport along $f$ agrees with the usual parallel transport along $f=\left\{f_{t}\right\}$ for the connection given by the transgression map, viewing $f$ as a path $\left\{f_{t}=\left.f\right|_{M \times\{t\}}\right\}$ in the space of maps from $M$ to $X$.

For 2-dimensional manifolds with boundary, the concept of the higherdimensional parallel transport was considered by Gawedzki in his pioneering paper [Ga] using transgression maps, and was developed by Brylinski in his fundamental work [B1] using differential geometry of gerbes. This paper owes much to the ideas in their works, and extends to any dimensional manifolds with boundary. Since the higher-dimensional parallel transports are given as special cases of more general formulas for the transgression maps, it seems an interesting problem to interpret our result in terms of differential geometry of higher gerbes $([\mathrm{B} 2],[\mathrm{BM}],[\mathrm{CMW}])$. For the geometry of gerbes see, in addition to Brylinski [B1], the excellent paper [H] by Hitchin. Carey-Mickelsson-Murray $[\mathrm{CMM}]$ give a geometric interpretation by bundle gerbes of higher holonomies for closed 2-manifolds, extending their previous impressive work [CM] for simple

Received April 10, 2000.

* This author's research is supported by Research Fellowship of the Japan Society for the Promotion of Science for Young Scientists. 
2-manifolds such as spheres or cylinders. In the interesting paper [F] Freed computes the derivative of the exponentiated $\eta$-invariant, considered as a section of the determinant bundle, with respect to the natural connection on the determinant bundle, which is important to this paper. In unpublished work, he has also developed an integration theory of Deligne cocycles, independently from and before us. Gajer $[\mathrm{G}]$ shows that the isomorphism [E] between smooth Deligne cohomology group and Cheeger-Simons' group of differential characters [CS] is given by 'holonomies' in the differential geometry of principal $B^{n} \mathbb{C}^{*}$-bundles, geometrically.

\section{Transgression maps}

In this section, we define the transgression maps on smooth Deligne cohomology and state their main properties (see $[\mathrm{GT}]$ ).

The smooth Deligne cohomology of a smooth manifold $X$ is defined to be the hypercohomology of the complex of sheaves

$$
\underline{\mathbb{C}}_{X}^{*} \stackrel{d \log }{\rightarrow} \underline{A}_{X}^{1} \stackrel{d}{\rightarrow} \cdots \stackrel{d}{\rightarrow} \underline{A}_{X}^{p},
$$

where $\underline{A}_{X}^{k}$ denotes the sheaf of smooth complex-valued $k$-forms on $X$ ([B1]).

The hypercohomology of a complex of sheaves is given with a Cech definition as follows. For any open covering $\mathfrak{U}$ of $X$ and any complex of sheaves $\mathcal{F}^{\bullet}$ the cochains $C^{\bullet}\left(\mathfrak{U}, \mathcal{F}^{\bullet}\right)$ form a double complex, one differential $\delta$ coming from the covering and another from the complex of sheaves $\mathcal{F}^{\bullet}$. The Čech hypercohomology groups is defined by

$$
H^{q}\left(X, \mathcal{F}^{\bullet}\right)=\underset{\mathfrak{U}}{\lim _{\longrightarrow}} H^{q}\left(C^{\bullet}\left(\mathfrak{U}, \mathcal{F}^{\bullet}\right)\right)
$$

where $H^{q}\left(C^{\bullet}\left(\mathfrak{U}, \mathcal{F}^{\bullet}\right)\right)$ denotes the cohomology of the single complex with differential, denoted by $D$, associated in the usual way to the double complex.

Next, we recall an important operation, called the transgression map on differential forms, in differential geometry on the space of smooth maps between smooth manifolds. Let $M$ be a compact oriented smooth manifold of dimension $m$ and $X$ be a closed smooth manifold. Let $\mathcal{M}=C^{\infty}(M, X)$ denote the space of all smooth maps $M \rightarrow X$ and ev $: \mathcal{M} \times M \rightarrow X$ denote the evaluation map defined by $\operatorname{ev}(f, m)=f(m)$ for $f \in \mathcal{M}$ and $m \in M$. The following diagram

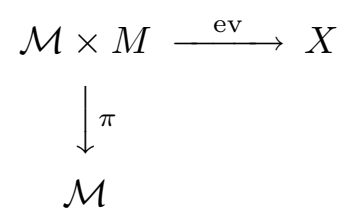

gives the transgression map on differential forms

$$
\bar{\psi}: A^{p}(X) \rightarrow A^{p-m}(\mathcal{M})
$$

defined by $\bar{\psi}(\omega)=\int_{M} \operatorname{ev}^{*} \omega$ for a differential form $\omega \in A^{p}(X)$, where $\int_{M}$ is the fiber integration along the fiber $M$ of the product bundle $\pi: \mathcal{M} \times M \rightarrow \mathcal{M}$. 
Now, as a refinement of this map, we define the transgression map on smooth Deligne cochains for each open covering of $X$. Let $\mathfrak{U}=\left\{U_{\alpha}\right\}_{\alpha \in I}$ be an open covering of $X$. An open covering $\mathfrak{V}$ of $\mathcal{M}$ is associated with $\mathfrak{U}$ as follows: for any pair $\beta=(K, \phi)$ of a triangulation $K$ of $M$ and a map $\phi: K \rightarrow I$, the open set $V_{\beta}$ of $\mathcal{M}$ is defined by

$$
V_{\beta}=\left\{f: M \rightarrow X \mid f(\sigma) \subset U_{\phi(\sigma)}, \sigma \in K\right\} .
$$

The set $\mathfrak{V}=\left\{V_{\beta}\right\}_{\beta \in J}$ of open sets is an open covering of $\mathcal{M}$, where $J=$ $\coprod_{K} \operatorname{Map}(\mathrm{K}, \mathrm{I})$.

We define the set of flags of simplices $F_{K}(i)$ by

$$
F_{K}(i)=\left\{\vec{\sigma}=\left(\sigma^{m-i}, \ldots, \sigma^{m}\right) \mid \sigma^{p} \in K, \operatorname{dim} \sigma^{p}=p, \sigma^{m-i} \subset \cdots \subset \sigma^{m}\right\} .
$$

and the set of sequences of integers $P_{q}(i)$ by

$$
P_{q}(i)=\left\{\vec{n}=\left(n_{1}, \ldots, n_{q}\right) \mid n_{j} \in \mathbb{Z}, m \geq n_{1} \geq \cdots \geq n_{q} \geq m-i\right\} .
$$

It is convenient to put $|\vec{n}|=\sum_{s=1}^{q} n_{s}$.

Definition 2.1. The transgression map associated with $\mathfrak{U}$

$\psi_{\mathfrak{U}}: C^{k}\left(\mathfrak{U}, \underline{\mathbb{C}}_{X}^{*} \stackrel{d \log }{\rightarrow} \underline{A}_{X}^{1} \rightarrow \cdots \rightarrow \underline{A}_{X}^{p}\right) \rightarrow C^{k-m}\left(\mathfrak{V}, \underline{\mathbb{C}}_{\mathcal{M}}^{*} \stackrel{d \log }{\rightarrow} \underline{A}_{\mathcal{M}}^{1} \rightarrow \cdots \rightarrow \underline{A}_{\mathcal{M}}^{p-m}\right)$

is defined by

$$
\psi_{\mathfrak{U}}\left(g, \omega^{1}, \ldots, \omega^{k}\right)=\left(h, \theta^{1}, \ldots, \theta^{k-m}\right),
$$

where

$$
\begin{aligned}
& h_{\beta_{0} \cdots \beta_{k-m}}=
\end{aligned}
$$

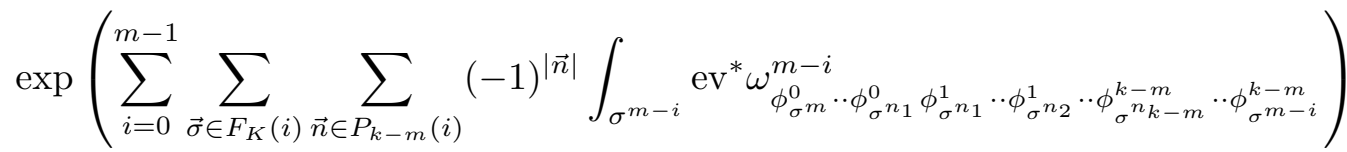

$$
\begin{aligned}
& \times \prod_{\vec{\sigma} \in F_{K}(m)} \prod_{\vec{n} \in P_{k-m}(m)}\left(\int_{\sigma^{0}} \mathrm{ev}^{*} g_{\phi_{\sigma}^{0} \cdot \cdot \phi_{\sigma}^{0} n_{1} \phi_{\sigma}^{1} n_{1} \cdot \cdot \phi_{\sigma}^{1} n_{2} \cdots \phi_{\sigma^{n}-m}^{k-m} \cdot \cdot \phi_{\sigma^{0}}^{k-m}}\right)^{(-1)^{|\vec{n}|}},
\end{aligned}
$$

Here, for $\beta_{0}=\left(K_{0}, \phi_{0}\right), \ldots, \beta_{r}=\left(K_{r}, \phi_{r}\right)$ we use a simplex $K$ which is a common subdivision of $K_{0}, \ldots, K_{r}$ and define the index $\phi_{\sigma^{j}}^{i} \in I$ to be $\phi_{\sigma^{j}}^{i}=\phi_{i} \circ \iota_{i}\left(\sigma^{j}\right)$, where $\iota_{i}(\sigma)$ for each simplex $\sigma \in K$ is the simplex in $K_{i}$ of smallest dimension such that $\sigma \subset \iota_{i}(\sigma)$. It is easy to see that the definition is independent of the choice of common subdivision.

Remark. We note that an orientation of $M$ induces that of $\sigma^{q}$ for $q=m-$ $i, \cdots, m$ along the flag $\sigma^{m-i} \subset \cdots \subset \sigma^{m}$. In particular, the induced orientation of $\sigma^{m-i}$ gives integration $\int_{\sigma^{m-i}}$. 
The following theorem is fundamental in the sequel.

Theorem 2.1. The transgression map $\psi_{\mathfrak{U}}$ satisfies the following:

$$
\psi_{\mathfrak{U}} \circ D=(-1)^{m} D \circ \psi_{\mathfrak{U}}+r^{*} \circ(\partial \psi)_{\mathfrak{U}},
$$

where $r: C^{\infty}(M, X) \rightarrow C^{\infty}(\partial M, X)$ is the restriction map and $(\partial \psi)_{\mathfrak{U}}$ is the transgression map associated with $\mathfrak{U}$ for the boundary $\partial M$.

Proof 1. The proof is essentially identical to that of Theorem 3.1 in [GT], except that here we consider all triangulations of $M$ at the same time, and the theorem follows by Stokes' theorem for fiber integration on differential forms.

Corollary 2.2. If the manifold $M$ has no boundary, then the map $\psi_{\mathfrak{U}}$ is a chain map.

Therefore the transgression map $\psi_{\mathfrak{U}}$ gives a map on cohomology

$$
\begin{aligned}
\psi_{\mathfrak{U}}^{\#}: H^{k}\left(\mathfrak{U}, \underline{\mathbb{C}}_{X}^{*} \stackrel{d \log }{\rightarrow} \underline{A}_{X}^{1} \rightarrow\right. \\
\left.\quad \rightarrow \cdots \rightarrow \underline{A}_{X}^{p}\right) \rightarrow H^{k-m}\left(\mathfrak{V}, \underline{\mathbb{C}}_{\mathcal{M}}^{*} \stackrel{d \log }{\rightarrow} \underline{A}_{\mathcal{M}}^{1} \rightarrow \cdots \rightarrow \underline{A}_{\mathcal{M}}^{p-m}\right) .
\end{aligned}
$$

Proposition 2.3. The homomorphisms $\left\{\psi_{\mathfrak{U}}^{\#}\right\}_{\mathfrak{U}}$ induces the direct limit

$$
\begin{aligned}
& \psi^{\#}: H^{k}\left(X, \underline{\mathbb{C}}_{X}^{*} \stackrel{d \log }{\rightarrow} \underline{A}_{X}^{1} \rightarrow\right. \\
&\left.\rightarrow \cdots \rightarrow \underline{A}_{X}^{p}\right) \rightarrow H^{k-m}\left(\mathcal{M}, \underline{\mathbb{C}}_{\mathcal{M}}^{*} \stackrel{d \log }{\rightarrow} \underline{A}_{\mathcal{M}}^{1} \rightarrow \cdots \rightarrow \underline{A}_{\mathcal{M}}^{p-m}\right) .
\end{aligned}
$$

Proof 2. Let $\mathfrak{U}^{\prime}=\left\{U_{\alpha^{\prime}}\right\}_{\alpha^{\prime} \in I^{\prime}}$ be a refinement of a cover $\mathfrak{U}=\left\{U_{\alpha}\right\}_{\alpha \in I}$ of $X$ with a map $\tau: I^{\prime} \rightarrow I$ such that $U_{\alpha^{\prime}}^{\prime} \subset U_{\tau\left(\alpha^{\prime}\right)}$. Let $\mathfrak{V}$ and $\mathfrak{V}^{\prime}$ be coverings of $\mathcal{M}$ associated with $\mathfrak{U}$ and $\mathfrak{U}^{\prime}$ indexed by $J=\coprod_{K} \operatorname{Map}(\mathrm{K}, \mathrm{I})$ and $J^{\prime}=\coprod_{K} \operatorname{Map}\left(\mathrm{K}, \mathrm{I}^{\prime}\right)$ respectively. Then $\mathfrak{V}^{\prime}$ is a refinement of $\mathfrak{V}$ with the map $\tilde{\tau}: J^{\prime} \rightarrow J$ defined by $\tilde{\tau}\left(\phi^{\prime}\right)=\tau \circ \phi^{\prime}$ for $\phi^{\prime} \in \operatorname{Map}\left(\mathrm{K}, \mathrm{I}^{\prime}\right)$. Proposition 2.3 follows from the commutative diagram

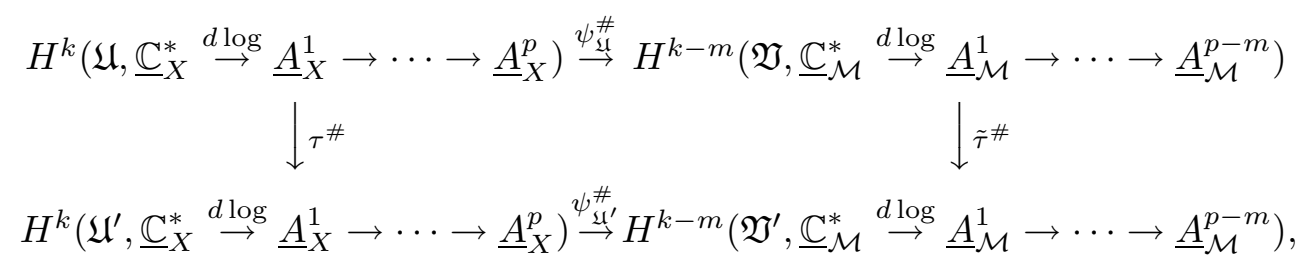

where $\tau^{\#}$ and $\tilde{\tau}^{\#}$ are homomorphisms induced in cohomology from maps $\tau$ and $\tilde{\tau}$ as with usual.

\section{Line bundles with connection}

Let $Y$ be a smooth manifold. The group of isomorphism classes of line bundles over $Y$ with connection is identified with the smooth Deligne cohomology group $H^{1}\left(Y, \mathbb{C}_{Y}^{*} \stackrel{d \log }{\rightarrow} \underline{A}_{Y}^{1}\right)$ as follows $([\mathrm{D}],[\mathrm{K}])$ : let $(L, \nabla)$ be a pair of a line bundle $L$ over $Y$ with a connection $\nabla$. Let $\left\{\left(U_{i}, s_{i}\right)\right\}_{i \in I}$ be a local system for the line bundle $L$, i.e. $\mathfrak{U}=\left\{U_{i}\right\}$ is an open covering of $Y$ and $s_{i}: U_{i} \rightarrow L$ is a nowhere 
vanishing smooth section on $U_{i}$, and $g_{i j}: U_{i} \cap U_{j} \rightarrow \mathbb{C}^{*}$ be the corresponding transition functions satisfying $s_{i}=g_{i j} s_{j}$. With the connection $\nabla$ we get a 1 -form $\theta_{i}=\nabla s_{i} / s_{i}$ on $U_{i}$. Then the cochain $\left(g_{i j}, \theta_{i}\right) \in C^{1}\left(\mathfrak{U}, \underline{\mathbb{C}}_{Y}^{*} \stackrel{d \log }{\longrightarrow} \underline{A}_{Y}^{1}\right)$ is a cocycle, and the cohomology class $\left[\left(g_{i j}, \theta_{i}\right)\right] \in H^{1}\left(\mathfrak{U}, \underline{\mathbb{C}}_{Y}^{*} \stackrel{d \log }{\rightarrow} \underline{A}_{Y}^{1}\right)$ is independent of the choice of local system. The map $[(L, \nabla)] \mapsto\left[\left(g_{i j}, \theta_{i}\right)\right]$ gives the isomorphism between the group of isomorphism classes of line bundles over $Y$ with connection and the smooth Deligne cohomology group $H^{1}\left(Y, \mathbb{C}_{Y}^{*} \stackrel{d \log }{\rightarrow} \underline{A}_{Y}^{1}\right)$.

Hence the transgression map for the closed manifold $M$ of dimension $m$

$$
\psi^{\#}: H^{m+1}\left(X, \underline{\mathbb{C}}_{X}^{*} \stackrel{d \log }{\rightarrow} \underline{A}_{X}^{1} \rightarrow \cdots \rightarrow \underline{A}_{X}^{m+1}\right) \rightarrow H^{1}\left(\mathcal{M}, \underline{\mathbb{C}}_{\mathcal{M}}^{*} \stackrel{d \log }{\rightarrow} \underline{A}_{\mathcal{M}}^{1}\right)
$$

gives an isomorphism class $[(\mathcal{L}, \nabla)]$ of a line bundle with connection over $\mathcal{M}$ for a cohomology class $[c] \in H^{m+1}\left(X, \underline{\mathbb{C}}_{X}^{*} \stackrel{d \log }{\rightarrow} \underline{A}_{X}^{1} \rightarrow \cdots \rightarrow \underline{A}_{X}^{m+1}\right)$. More precisely, for cohomologous $(m+1)$-cocycles $c, c^{\prime}$ and an $m$-cochain $b$ satisfying $c-c^{\prime}=$ $D b$ we have line bundles with connection $(\mathcal{L}, \nabla),\left(\mathcal{L}^{\prime}, \nabla^{\prime}\right)$ and an isomorphism $\mu_{b}:(\mathcal{L}, \nabla) \rightarrow\left(\mathcal{L}^{\prime}, \nabla^{\prime}\right)$ between line bundles with connection.

Next we consider $(m+1)$-dimensional manifold $N$ with boundary $\partial N=M$. Let $\mathfrak{V}=\left\{V_{\beta}\right\}_{\beta \in J}$ and $\overline{\mathfrak{V}}=\left\{\bar{V}_{\bar{\beta}}\right\}_{\bar{\beta} \in \bar{J}}$ be the open coverings of $\mathcal{N}=C^{\infty}(N, X)$ and $\mathcal{M}=C^{\infty}(M, X)$ associated with an open covering $\mathfrak{U}=\left\{U_{\alpha}\right\}_{\alpha \in I}$ of $X$, respectively. For an $(m+1)$-cocycle $c \in C^{m+1}\left(\mathfrak{U}, \underline{\mathbb{C}}_{X}^{*} \stackrel{d \log }{\rightarrow} \underline{A}_{X}^{1} \rightarrow \cdots \rightarrow \underline{A}_{X}^{m+1}\right)$ we denote $\left(h_{\beta}\right)=(-1)^{m+1} \psi_{\mathfrak{U}}(c)$ and $\left(g_{\bar{\beta}_{0} \bar{\beta}_{1}}, \theta_{\bar{\beta}}\right)=(\partial \psi)_{\mathfrak{U}}(c)$. Then the line bundle $\mathcal{L}$ over $\mathcal{M}$ associated with $c$ has $\left\{g_{\bar{\beta}_{0} \bar{\beta}_{1}}\right\}$ as the transition functions. Let $r: \mathcal{N} \rightarrow \mathcal{M}$ be the restriction map $\left.f \mapsto f\right|_{M}$. The pullback $r^{*} \mathcal{L}$ over $\mathcal{N}$ is given by

$$
r^{*} \mathcal{L}=\coprod_{\beta \in J} V_{\beta} \times \mathbb{C} / \sim
$$

where the equivalence relation $\sim$ is given by declaring $(f, \lambda)_{\beta_{0}} \sim\left(f, \lambda^{\prime}\right)_{\beta_{1}}$ for $(f, \lambda)_{\beta_{0}} \in V_{\beta_{0}} \times \mathbb{C}$ and $\left(f, \lambda^{\prime}\right)_{\beta_{1}} \in V_{\beta_{1}} \times \mathbb{C}$ if $\lambda=g_{\bar{\beta}_{0} \bar{\beta}_{1}}\left(\left.f\right|_{M}\right) \lambda^{\prime}$. We define a section $P_{c}: \mathcal{N} \rightarrow r^{*} \mathcal{L}$ of the line bundle $r^{*} \mathcal{L}$ by the equivalence class $P_{c}(f)=$ $\left[\left(f, h_{\beta}(f)\right)_{\beta}\right]$ for $f \in V_{\beta} \subset \mathcal{N}$.

Proposition 3.1. (i) The global section $P_{c}$ of the line bundle $r^{*} \mathcal{L}$ is welldefined.

(ii) For cohomologous $(m+1)$-cocycles $c, c^{\prime}$ and an $m$-cochain $b$ satisfying $c-$ $c^{\prime}=D b$, we have $P_{c}=\mu_{b}^{*}\left(P_{c^{\prime}}\right)$.

Proof 3. (i ) It suffices to show for $f \in V_{\beta_{0}} \cap V_{\beta_{1}}$

$$
h_{\beta_{0}}(f)=g_{\bar{\beta}_{0} \bar{\beta}_{1}}\left(\left.f\right|_{M}\right) h_{\beta_{1}}(f),
$$

which follows from Theorem 2.1 with $k=p=m+1$.

(ii) Let $\left(k_{\bar{\beta}}\right)=(-1)^{m}(\partial \psi)_{\mathfrak{U}}(b)$. Then, by Theorem 2.1 we have

$$
h_{\beta}^{\prime}(f)=k_{\bar{\beta}}\left(\left.f\right|_{M}\right) h_{\beta}(f)
$$

which shows (ii) since the isomorphism $\mu_{b}$ associated with the cochain $b$ gives the change of the factor $k_{\bar{\beta}}$ on an open set $U_{\beta}$ locally. 
To state the formula which computes the derivative of the section $P_{c}$ with the pullback connection $r^{*} \nabla$ by the restriction map $r$ of the connection $\nabla$, we recall that the morphism of complexes

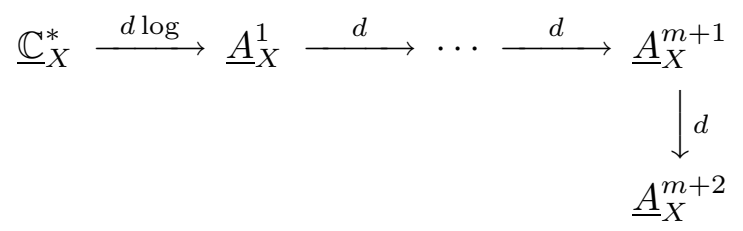

induces the homomorphism

$$
d: H^{m+1}\left(X, \underline{\mathbb{C}}_{X}^{*} \stackrel{d \log }{\rightarrow} \underline{A}_{X}^{1} \rightarrow \cdots \rightarrow \underline{A}_{X}^{m+1}\right) \rightarrow A^{m+2}(X),
$$

where $A^{m+2}(X)$ is the space of complex-valued $(m+2)$-forms. Let $\Omega=d([c])$. The following is an extension of a result for a 2-dimensional manifold by Brylinski [B1], obtained using differential geometry of gerbes, to any-dimensional manifold $N$.

Proposition 3.2. Let $\gamma=\left\{\gamma_{s}\right\}$ be a path in $\mathcal{N}$. Then, the section $P_{c}$ satisfies with the pullback connection $r^{*} \nabla$

$$
r^{*} \nabla_{\dot{\gamma}_{s}} P_{c}=\left(\int_{N} \iota \dot{\gamma}_{s} \Omega\right) P_{c}
$$

where $\iota_{\dot{\gamma}_{s}}: A^{m+2}(X) \rightarrow A^{m+1}(N)$ is the contraction along $\dot{\gamma}_{s} \in \Gamma\left(N, \gamma_{s}^{*} T X\right)$.

Proof 4. Locally on an open set $V_{\beta}$, we consider the function $h_{\beta}$ corresponding to the section $P_{c}$. Then by the definition of $h_{\beta},\left(h_{\beta}\right)=(-1)^{m+1} \psi_{\mathfrak{U}}(c)$, we have

$$
\begin{aligned}
& h_{\beta}^{-1} d h_{\beta} \\
& =\sum_{i=0}^{m} \sum_{\vec{\sigma} \in F(i)}(-1)^{m+1} d \int_{\sigma^{m+1-i}} \mathrm{ev}^{*} \omega_{\phi_{\sigma^{m+1}} \phi_{\sigma} m \ldots \phi_{\sigma^{m+1-i}}}^{m+1-i} \\
& +\sum_{\vec{\sigma} \in F(m+1)}(-1)^{m+1} \int_{\sigma^{0}} \mathrm{ev}^{*}\left(g^{-1} d g\right)_{\phi_{\sigma} m+1} \phi_{\sigma^{m}} \ldots \phi_{\sigma^{0}}
\end{aligned}
$$

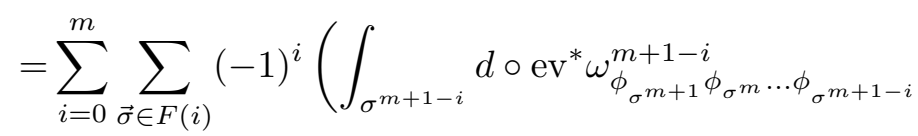

$$
\begin{aligned}
& \left.-\int_{\partial \sigma^{m+1-i}} \operatorname{ev}^{*} \omega_{\phi_{\sigma^{m+1}} \phi_{\sigma^{m} \cdots} \ldots \phi_{\sigma^{m+1-i}}}^{m+1-i}\right) \\
& +\sum_{\vec{\sigma} \in F(m+1)}(-1)^{m+1} \int_{\sigma^{0}} \mathrm{ev}^{*}\left(g^{-1} d g\right)_{\phi_{\sigma} m+1} \phi_{\sigma^{m}} \ldots \phi_{\sigma^{0}} \\
& =\int_{N} \mathrm{ev}^{*} \Omega-\sum_{i=1}^{m+1} \sum_{\vec{\sigma} \in F(i)} \\
& \int_{\sigma^{m+1-i}}\left(\operatorname{ev}^{*}\left(\delta \omega^{m+2-i}\right)_{\phi_{\sigma^{m+1}} \ldots \phi_{\sigma^{m+1-i}}}-(-1)^{i} \operatorname{ev}^{*} \omega_{\phi_{\sigma^{m+1}}^{m+2-i} \phi_{\sigma^{m+2-i}}}\right) \text {. }
\end{aligned}
$$


Since for each partial flag of simplices $\sigma^{m+1-i} \subset \cdots \subset \widehat{\sigma^{p}} \subset \cdots \subset \sigma^{m+1}$, $p=m+2-i, \ldots, m$, and $\sigma^{m+1-i} \subset \cdots \subset \sigma^{m} \subset \widehat{\sigma^{m+1}}$ which is not contained in $\partial N=M$ there are exactly two flags which are 'completions' of the partial flag and induce opposite orientations of $\sigma^{m+1-i}$, we have

$$
\begin{aligned}
& \sum_{\vec{\sigma} \in F(i)} \int_{\sigma^{m+1-i}} \mathrm{ev}^{*}\left(\delta \omega^{m+2-i}\right)_{\phi_{\sigma^{m+1}} \cdots \phi_{\sigma^{m+1-i}}}= \\
& \sum_{\vec{\sigma} \in F(i)} \int_{\sigma^{m+1-i}}(-1)^{i} \mathrm{ev}^{*} \omega_{\phi_{\sigma^{m+1}} \cdots \phi_{\sigma^{m+2-i}}}^{m+2-i} \\
& +\sum_{\vec{\sigma} \in \partial F(i-1)} \int_{\sigma^{m+1-i}} \mathrm{ev}^{*} \omega_{\phi_{\sigma^{m}} \ldots \phi_{\sigma^{m+1-i}}}^{m+2-i},
\end{aligned}
$$

where

$$
\partial F(i)=\left\{\vec{\sigma}=\left(\sigma^{m-i}, \ldots, \sigma^{m}\right) \mid \sigma^{p} \in K, \operatorname{dim} \sigma^{p}=p, \sigma^{m-i} \subset \cdots \subset \sigma^{m} \subset \partial N\right\} .
$$

Therefore,

$$
h_{\beta}^{-1} d h_{\beta}+\sum_{i=0}^{m} \sum_{\vec{\sigma} \in \partial F(i)} \int_{\sigma^{m-i}} \mathrm{ev}^{*} \omega_{\phi_{\sigma} \ldots \phi_{\sigma}-i}^{m+1-i}=\int_{N} \mathrm{ev}^{*} \Omega .
$$

Setting $h_{s}=h_{\beta}\left(\gamma_{s}\right)$, by the definition of the 1 -form $\theta^{1}$ which corresponds to the connection $r^{*} \nabla$,

$$
\frac{d h_{s}}{d s}+\left\langle\theta^{1}\left(\gamma_{s}\right), \dot{\gamma}_{s}\right\rangle h_{s}=\left(\int_{N} \iota_{\dot{\gamma}_{s}} \Omega\right) h_{s}
$$

which completes the proof.

More generally, let $N$ be a compact manifolds of dimension $m+1$ with boundary $\partial N=\coprod M_{i}$, where $M_{1}, \ldots, M_{s}$ are given the orientations opposite to that induced from $N$ and $M_{s+1}, \ldots, M_{t}$ are given the induced orientations. Let $\left(\mathcal{L}^{i}, \nabla^{i}\right)$ be the line bundle with connection over $\mathcal{M}_{i}=C^{\infty}\left(M_{i}, X\right)$ associated with $c \in C^{m+1}\left(\mathfrak{U}, \underline{\mathbb{C}}_{X}^{*} \stackrel{d \log }{\rightarrow} \underline{A}_{X}^{1} \rightarrow \cdots \rightarrow \underline{A}_{X}^{m+1}\right)$ and $r_{i}: \mathcal{N} \rightarrow \mathcal{M}_{i}$ be the restriction map $\left.f \mapsto f\right|_{M_{i}}$. Then by Proposition 3.1 we have an isomorphism of line bundles

$$
P_{c}: r_{1}^{*} \mathcal{L}^{1} \otimes \cdots \otimes r_{s}^{*} \mathcal{L}^{s} \rightarrow r_{s+1}^{*} \mathcal{L}^{s+1} \otimes \cdots \otimes r_{t}^{*} \mathcal{L}^{t}
$$

Therefore, for a map $f \in \mathcal{N}$ we have an isomorphism between fibers

$$
P_{c}(f): \mathcal{L}_{\left.f\right|_{M_{1}}}^{1} \otimes \cdots \otimes \mathcal{L}_{\left.f\right|_{M_{s}}}^{s} \rightarrow \mathcal{L}_{\left.f\right|_{M_{s+1}}}^{s+1} \otimes \cdots \otimes \mathcal{L}_{\left.f\right|_{M_{t}}}^{t}
$$

called the higher-dimensional parallel transport along a generalized path $f$. From the definition it is not difficult to see that the higher-dimensional parallel transport is invariant under any reparametrization of generalized paths, i.e. any diffeomorphism of $N$ which is identity on the boundary, and is compatible with gluing two $(m+1)$-manifolds with boundary along a common component of their boundary, i.e. a gluing of manifolds corresponds to a composition of higherdimensional parallel transports associated with these manifolds respectively. 
In particular, for $f: N=M \times[0,1] \rightarrow X$ we have an isomorphism

$$
P_{c}(f): \mathcal{L}_{f_{0}} \rightarrow \mathcal{L}_{f_{1}},
$$

where $f_{t}=\left.f\right|_{M \times\{t\}}$. On the other hand, considering $f$ as a path $\left\{f_{t}\right\}$ in $\mathcal{M}$, we have the usual parallel transport along the path $f=\left\{f_{t}\right\}$ for the line bundle with connection $(\mathcal{L}, \nabla)$ over $\mathcal{M}$

$$
P_{c}^{\prime}(f): \mathcal{L}_{f_{0}} \rightarrow \mathcal{L}_{f_{1}}
$$

Theorem 3.3. For $N=M \times[0,1]$ the higher-dimensional parallel transport $P_{c}$ agrees with the usual parallel transport $P_{c}^{\prime}$.

Proof 5. For $f: M \times[0,1] \rightarrow X$ let $\gamma=\left\{\gamma_{s}\right\}$ be a path in $\mathcal{N}$ given by

$$
\gamma_{s}(m, t)=f(m, s t) \text {. }
$$

Then by Proposition 3.2

$$
\begin{aligned}
\nabla_{\dot{f}_{s}} P_{c}\left(f_{s}\right) & =r^{*} \nabla_{\dot{\gamma}_{s}} P_{c}\left(\gamma_{s}\right) \\
& =\left(\int_{N} \iota_{\dot{\gamma}_{s}} \Omega\right) P_{c}\left(\gamma_{s}\right) .
\end{aligned}
$$

Since $\cup_{s} \operatorname{Im} \gamma_{s}$ is not $(m+2)$-dimensional but $(m+1)$-dimensional,

$$
\int_{N} \iota_{\dot{\gamma}_{s}} \Omega=0
$$

which completes the proof of Theorem 3.3.

Finally we note that for general manifold with boundary the higher-dimensional parallel transport can not be given by any differential equation since general manifolds have no 'time' parameter globally.

\section{Acknowledgments}

We are grateful to Prof. Brylinski for valuable advice and, in particular, for his suggestion that our method in $[\mathrm{GT}]$ can be applied to this subject. We are also grateful to Prof. Kori for useful discussions and for sending his stimulating preprint $[\mathrm{K}]$, and to Prof. Kohno for helpful suggestions and constant encouragement.

\section{References}

[B1] J.-L. Brylinski, Loop spaces, characteristic classes and geometric quantization, Progress in Mathematics 107, Birkhäuser Boston, Inc., Boston, MA, 1993.

[B2] - Geometric construction of Quillen line bundles, Advances in geometry, 107146, Progr. Math. 172, Birkhäuser Boston, Boston, MA, 1999.

[BM] J.-L. Brylinski and D. McLaughlin, The geometry of degree-4 characteristic classes and of line bundles on loop spaces. II, Duke Math. J. 83 (1996), 105-139.

[CM] A. Carey and M. K. Murray, Holonomy and the Wess-Zumino term, Lett. Math. Phys. 12 (1986), 323-327.

[CMM] A. Carey, J. Mickelsson, and M. Murray, Bundle gerbes applied to quantum field theory, Rev. Math. Phys. 12 (2000), 65-90. 
[CMW] A. Carey, M. Murray, and B. Wang, Higher bundle gerbes and cohomology classes in gauge theories, J. Geom. Phys. 21 (1997), 183-197.

[CS] J. Cheeger and J. Simons, Differential characters and geometric invariants, Geometry and topology, 50-80, Lecture Notes in Math. 1167, Springer, Berlin-New York, 1985.

[D] P. Deligne, Le symbole modéré, Inst. Hautes tudes Sci. Publ. Math. 73 (1991), 147181.

[E] H. Esnault, Characteristic classes of flat bundles, Topology 27 (1988), 323-352.

[F] D. Freed, Determinant line bundles revisited, Geometry and physics (Aarhus, 1995), 187-195, Lecture Notes in Pure and Appl. Math. 184, Dekker, New York, 1997.

[G] P. Gajer, Higher holonomies, geometric loop groups and smooth Deligne cohomology, Advances in geometry, 195-235, Progr. Math. 172, Birkhäuser Boston, Boston, MA, 1999.

[Ga] K. Gawedzki, Topological actions in two-dimensional quantum field theories, Nonperturbative quantum field theory (Cargèse, 1987), 101-141, NATO Adv. Sci. Inst. Ser. B: Phys. 185, Plenum, New York-London, 1988.

[GT] K. Gomi, Y. Terashima, A fiber integration formula for the smooth Deligne cohomology, Internat. Math. Res. Notices 13 (2000), 699-708.

[H] N. Hitchin, Lectures on special lagrangian submanifolds, math.DG/9907034

[K] T. Kori, 4-dimensional Wess-Zumino-Witten model and central extensions of $\Omega^{3} G$, preprint.

[Ko] B. Kostant, Quantization and unitary representations. I. Prequantization, Lectures in modern analysis and applications, III, pp. 87-208. Lecture Notes in Math. 170, Springer, Berlin, 1970.

Graduate School of Mathematical Sciences, University of Tokyo, Komaba 3-8-1 MEguro-KU, TOKYO, 153-8914 JAPAN.

E-mail address: kgomi@ms.u-tokyo.ac.jp

E-mail address: tera@ms.u-tokyo.ac.jp 